\title{
Erratum to: On Estimation and Selection of Autologistic Regression Models via Penalized Pseudolikelihood
}

\author{
Rao Fu, Andrew L. Thurman, Tingjin Chu, Michelle M. SteEn-ADAmS, \\ and Jun ZHU
}

\section{ERRATUM TO: JABES DOI 10.1007/S13253-013-0144-Z}

\section{CORRECTED TEXT}

1. Original text in Sect. 4.1, paragraph 1

When the sample size is smaller $(n=225)$, the average number of correctly identified zero (and non-zero) regression coefficients is largely closer to the truth when $\eta$ is smaller corresponding to weaker spatial dependence. For the larger sample $(n=$ 900), the effect of $\eta$ is not as obvious. For either sample size, there is no apparent effect of $\beta_{1}$ (i.e., large-scale structure) on the average number of correctly identified zero or non-zero regression coefficients.

Corrected text in Sect. 4.1, paragraph 1

For either sample size, there is no apparent effects of $\beta_{1}$ and $\eta$ on the average number of correctly identified zero or non-zero regression coefficients.

2. Original text in Sect. 5, paragraph 4

For the two centered models, the same sets of covariates were chosen, and for the two uncentered models, nearly the same sets were chosen.

Corrected text in Sect. 5, paragraph 4

Nearly the same sets of covariates were chosen for the two uncentered models and similarly, for the two centered models.

The online version of the original article can be found under doi:10.1007/s13253-013-0144-z.

Rao Fu and Andrew L. Thurman, Department of Statistics, University of Wisconsin, Madison, WI 53706, USA (E-mail: rfu7@wisc.edu, E-mail: athurman@wisc.edu). Tingjin Chu, School of Statistics, Renmin University, Beijing 100872, China (E-mail: chutj03@ruc.edu.cn). Michelle M. Steen-Adams, Department of Environmental Studies, University of New England, Biddeford, ME 04005, USA (E-mail: msteenadams@une.edu).

Jun Zhu $(\bowtie)$, Department of Statistics and Department of Entomology, University of Wisconsin, Madison, WI 53706, USA (E-mail: jzhu@stat.wisc.edu). 
3. Original text in Sect. 5, paragraph 6

The interaction variable, PolyPr $\times$ MxPolyPr, was selected in all four cases, indicating a positive association with APB.

Corrected text in Sect. 5, paragraph 6

The interaction variable, $\mathrm{PolyPr} \times \mathrm{MxPolyPr}$, was selected in three of the four cases, indicating a positive association with APB.

\section{CORRECTED FIGURES AND TABLES}

See Tables 1, 2, 3 and also see Figs. 2, 3, 4 and 5.

Corrections of original Tables 1, 2 and 3 are in bold.

Table 1. Average number of correctly identified non-zero and zero regression coefficients by adaptive Lasso (AL) or smoothly clipped absolute deviation (SCAD) when $\beta_{1}=5$ (weak large-scale structure) for uncentered and centered model, sample size $n=225$ or 900 , and antoregression coefficient $\eta=0.3$ or 0.7 .

\begin{tabular}{|c|c|c|c|c|c|c|c|c|c|}
\hline \multirow[t]{3}{*}{ Model } & \multirow{3}{*}{$\begin{array}{c}\left\{\beta_{j}\right\} \\
n\end{array}$} & \multicolumn{4}{|c|}{ Number of non-zero estimates } & \multicolumn{4}{|c|}{ Number of zero estimates } \\
\hline & & \multicolumn{2}{|c|}{$\eta=0.3$} & \multicolumn{2}{|c|}{$\eta=0.7$} & \multicolumn{2}{|c|}{$\eta=0.3$} & \multicolumn{2}{|c|}{$\eta=0.7$} \\
\hline & & $\mathrm{AL}$ & SCAD & $\mathrm{AL}$ & SCAD & $\mathrm{AL}$ & SCAD & $\mathrm{AL}$ & SCAD \\
\hline \multirow[t]{2}{*}{ Uncentered } & 225 & 2.83 & 2.89 & 2.74 & 2.90 & 6.37 & 5.98 & 6.59 & 5.74 \\
\hline & 900 & 3.00 & 3.00 & 3.00 & 3.00 & 6.81 & 5.95 & 6.88 & 6.19 \\
\hline \multirow[t]{2}{*}{ Centered } & 225 & 2.70 & 2.85 & 2.75 & 2.87 & 5.92 & 4.87 & 6.26 & 4.74 \\
\hline & 900 & 3.00 & 3.00 & 3.00 & 3.00 & 6.89 & 5.74 & 6.82 & 5.59 \\
\hline
\end{tabular}

Table 2. Average number of correctly identified non-zero and zero regression coefficients by adaptive Lasso (AL) or smoothly clipped absolute deviation (SCAD) when $\beta_{1}=1$ (strong large-scale structure) for uncentered and centered model, sample size $n=225$ or 900 , and antoregression coefficient $\eta=0.3$ or 0.7 .

\begin{tabular}{|c|c|c|c|c|c|c|c|c|c|}
\hline \multirow[t]{3}{*}{ Model } & \multirow{3}{*}{$\begin{array}{c}\left\{\beta_{j}\right\} \\
n\end{array}$} & \multicolumn{4}{|c|}{ Number of non-zero estimates } & \multicolumn{4}{|c|}{ Number of zero estimates } \\
\hline & & \multicolumn{2}{|c|}{$\eta=0.3$} & \multicolumn{2}{|c|}{$\eta=0.7$} & \multicolumn{2}{|c|}{$\eta=0.3$} & \multicolumn{2}{|c|}{$\eta=0.7$} \\
\hline & & $\mathrm{AL}$ & SCAD & AL & SCAD & $\mathrm{AL}$ & SCAD & $\mathrm{AL}$ & SCAD \\
\hline \multirow[t]{2}{*}{ Uncentered } & 225 & 3.00 & 3.00 & 2.86 & 2.99 & 6.57 & 5.61 & 6.53 & 5.55 \\
\hline & 900 & 3.00 & 3.00 & 3.00 & 3.00 & 6.88 & 5.69 & 6.95 & 6.43 \\
\hline \multirow[t]{2}{*}{ Centered } & 225 & 2.98 & 3.00 & 2.96 & 2.99 & 6.60 & 5.03 & 6.54 & 4.95 \\
\hline & 900 & 3.00 & 3.00 & 3.00 & 3.00 & 6.85 & 5.30 & 6.94 & 5.18 \\
\hline
\end{tabular}


Table 3. Maximum penalized pseudolikelihood estimates (MPPLE) of the regression coefficients and the autoregression coefficient $\eta$ for uncentered or centered models and $0-1$ or \pm 1 coding of the response variable. The standard errors of MPPLE of the regression coefficients and the autoregression coefficient are given in the parentheses.

\begin{tabular}{|c|c|c|c|c|}
\hline \multirow{2}{*}{ Covariates } & \multicolumn{2}{|c|}{$0-1$ coding } & \multicolumn{2}{|c|}{ \pm 1 coding } \\
\hline & Uncentered & Centered & Uncentered & Centered \\
\hline Reserv & $2.90(1.528)$ & $1.58(0.500)$ & $1.72(1.026)$ & $1.21(0.354)$ \\
\hline PolyNm & - & - & - & - \\
\hline PolyPr & - & - & - & - \\
\hline MxPolyPr & - & - & - & - \\
\hline $\log ($ TotOwn $)$ & $0.68(0.374)$ & - & $0.34(0.151)$ & - \\
\hline AvParcl & $-0.44(0.356)$ & - & - & - \\
\hline Reserv $\times$ PolyNm & $-0.38(0.522)$ & - & $-0.24(0.229)$ & - \\
\hline Reserv×PolyPr & $-0.67(0.319)$ & - & $-0.42(0.208)$ & - \\
\hline Reserv $\times$ MxPolyPr & - & - & - & - \\
\hline Reserv $\times \log ($ TotOwn) & - & - & - & - \\
\hline Reserv×AvParcl & - & - & - & - \\
\hline PolyNm×PolyPr & $0.04(0.067)$ & - & - & - \\
\hline PolyNm×MxPolyPr & - & - & - & - \\
\hline PolyNm× log (TotOwn) & $0.03(0.220)$ & - & - & - \\
\hline PolyNm×AvParcl & $0.35(0.264)$ & - & $0.02(0.063)$ & - \\
\hline PolyPrxMxPolyPr & $0.83(0.271)$ & - & $0.33(0.167)$ & $0.26(0.058)$ \\
\hline PolyPrx log (TotOwn) & $-0.65(0.588)$ & - & $-0.35(0.235)$ & - \\
\hline PolyPrxAvParcl & - & - & - & - \\
\hline MxPolyPrx log (TotOwn) & - & - & - & - \\
\hline MxPolyPr×AvParcl & - & - & - & - \\
\hline $\log ($ TotOwn $) \times$ AvParcl & - & - & - & - \\
\hline$\eta$ & $1.22(0.089)$ & $1.39(0.048)$ & $0.33(0.045)$ & $0.35(0.016)$ \\
\hline
\end{tabular}


(a)

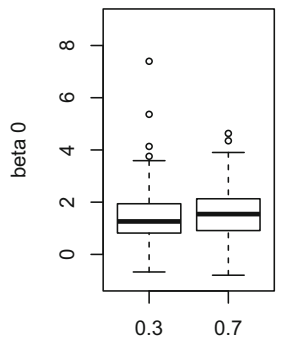

eta
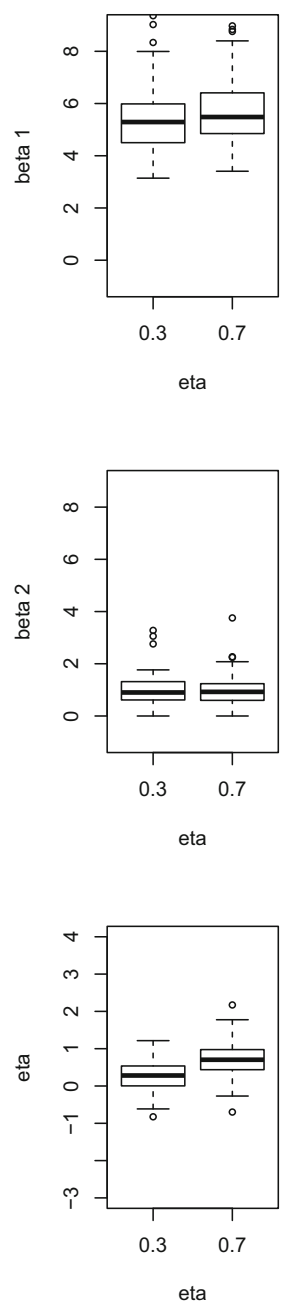

(b)
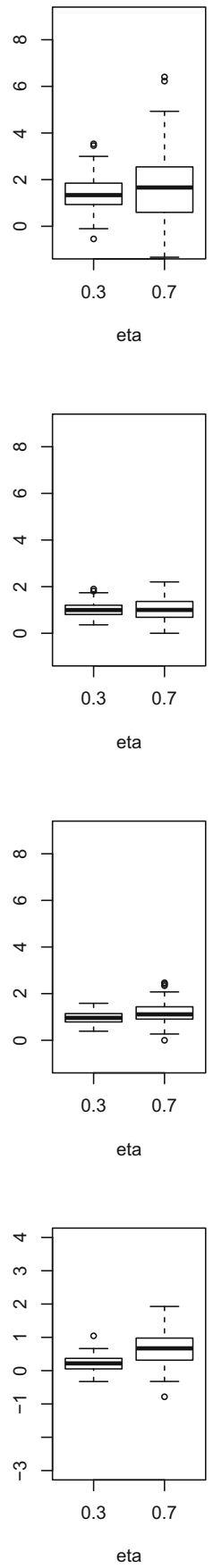

(c)

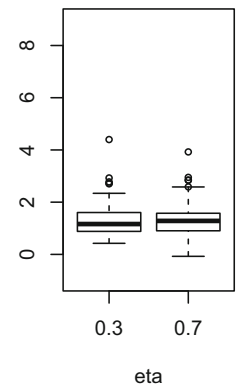

eta
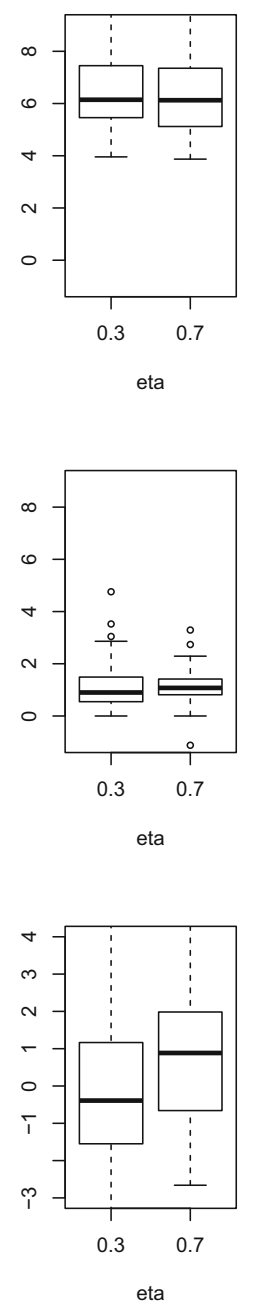

(d)
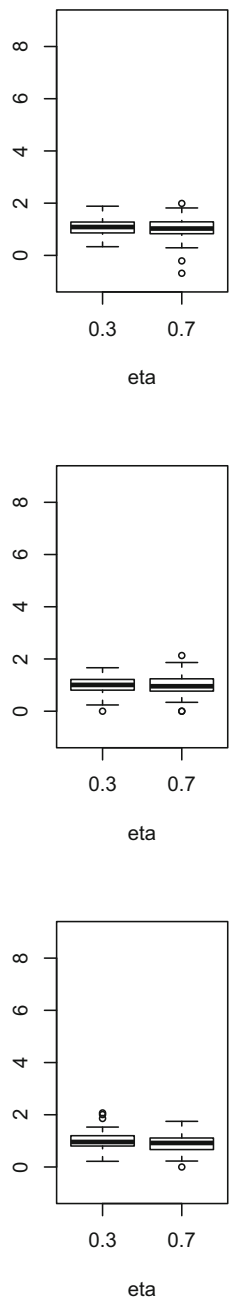

eta

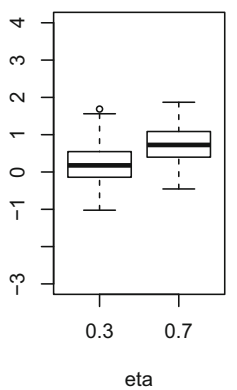

Figure 2. Box plot of the MPPLE $\widehat{\beta}_{0}$ (row 1), $\widehat{\beta}_{1}$ (row 2), $\widehat{\beta}_{2}$ (row 3 ), and $\widehat{\eta}$ (row 4 ) from the 100 simulations with sample size $n=225$. Column (a): uncentered model with $\beta_{1}=5$ (weak large-scale structure); (b): uncentered model with $\beta_{1}=1$ (strong large-scale structure); (c): centered model with $\beta_{1}=5$ (weak large-scale structure); (d): centered model with $\beta_{1}=1$ (strong large-scale structure). The true values are $\beta_{0}=1, \beta_{1}=1$ or $5, \beta_{2}=1$, $\beta_{3}=1$, and $\eta=0.3$ or 0.7 . The box plot of $\widehat{\beta}_{3}$ is similar to $\widehat{\beta}_{2}$ and omitted to save space. 
(a)
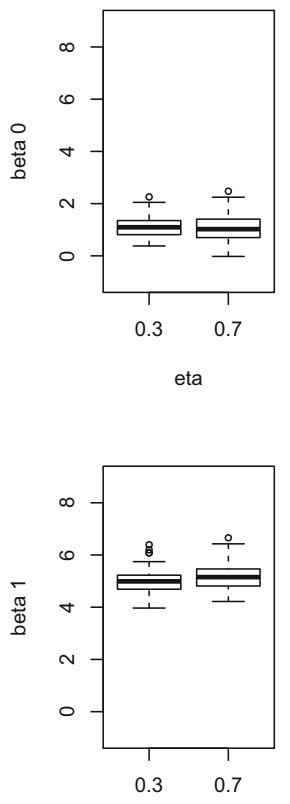

eta
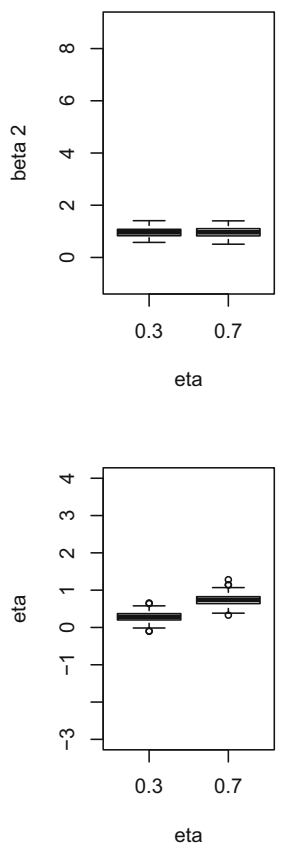

(b)
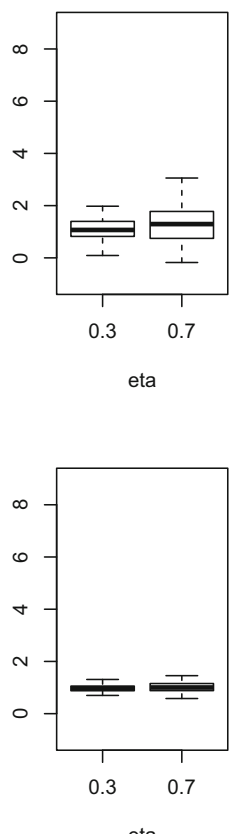

eta
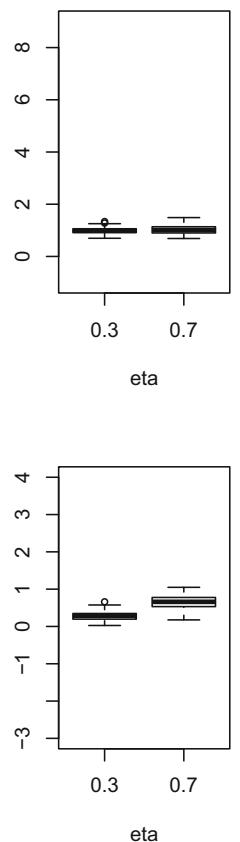

(c)

(d)
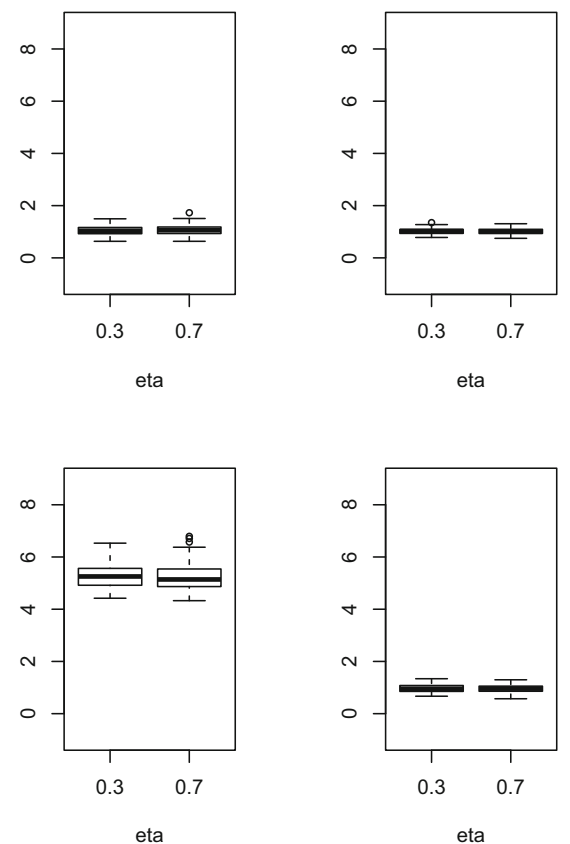

eta
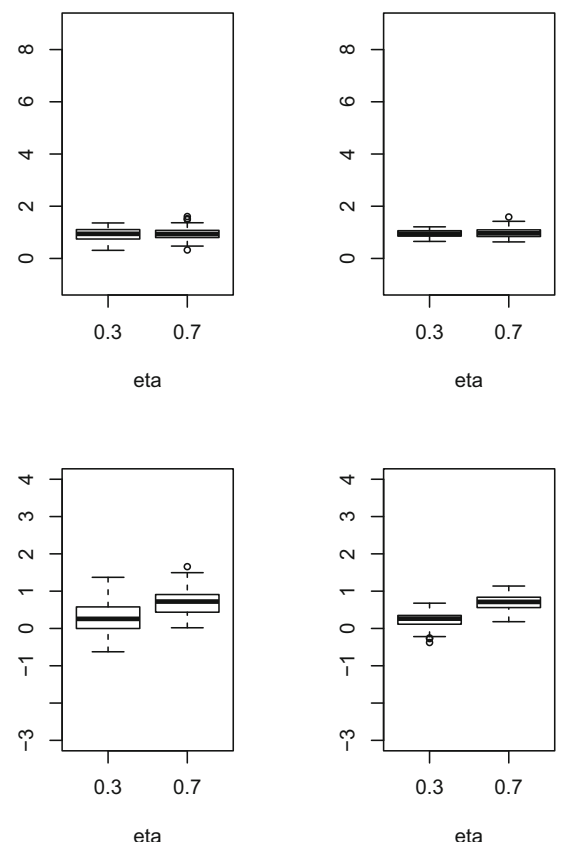

Figure 3. Box plot of the MPPLE $\widehat{\beta}_{0}$ (row 1), $\widehat{\beta}_{1}$ (row 2), $\widehat{\beta}_{2}$ (row 3), and $\widehat{\eta}$ (row 4) from the 100 simulations with sample size $n=900$. Column (a): uncentered model with $\beta_{1}=5$ (weak large-scale structure); (b): uncentered model with $\beta_{1}=1$ (strong large-scale structure); (c): centered model with $\beta_{1}=5$ (weak large-scale structure); (d): centered model with $\beta_{1}=1$ (strong large-scale structure). The true values are $\beta_{0}=1, \beta_{1}=1$ or $5, \beta_{2}=1$, $\beta_{3}=1$, and $\eta=0.3$ or 0.7 . The box plot of $\widehat{\beta}_{3}$ is similar to $\widehat{\beta}_{2}$ and omitted to save space. 
(a)
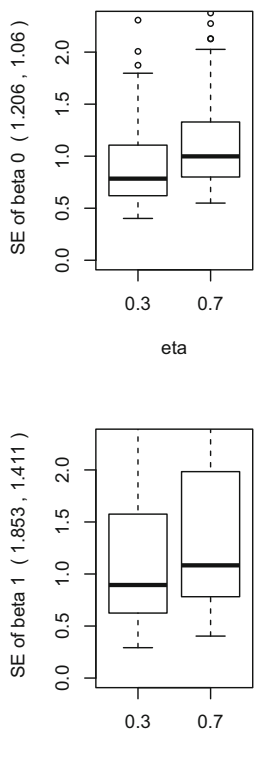

eta
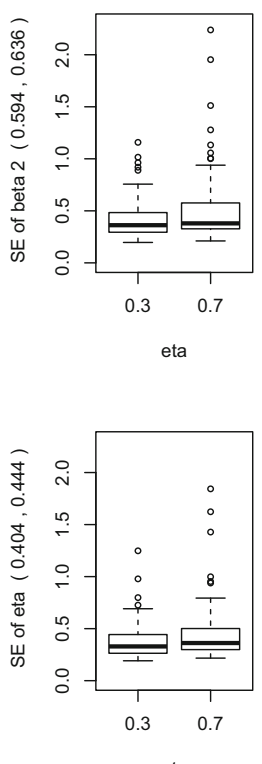

(b)
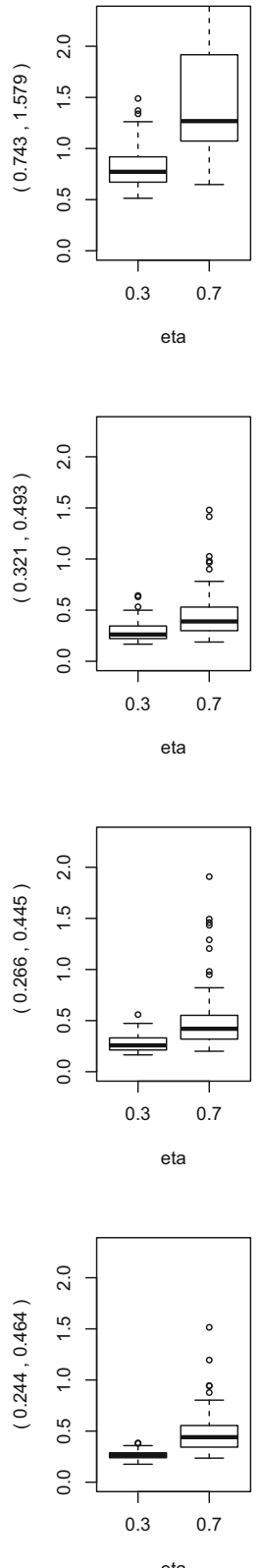

(c)
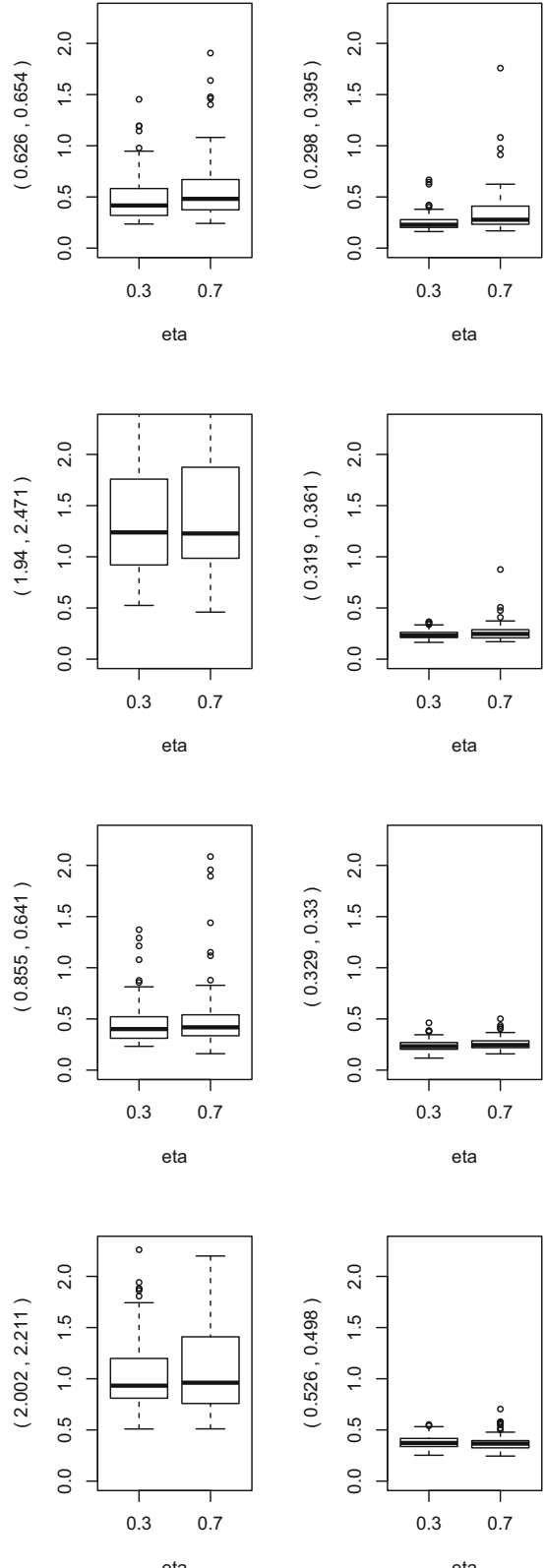

Figure 4. Box plot of the standard error of $\widehat{\beta}_{0}$ (row 1), $\widehat{\beta}_{1}$ (row 2), $\widehat{\beta}_{2}$ (row 3), and $\widehat{\eta}$ (row 4) from the 100 simulations with sample size $n=225$. Column (a): uncentered model with $\beta_{1}=5$ (weak large-scale structure); (b): uncentered model with $\beta_{1}=1$ (strong large-scale structure); (c): centered model with $\beta_{1}=5$ (weak largescale structure); (d): centered model with $\beta_{1}=1$ (strong large-scale structure). The nominal standard error of the MPPLE from the 100 simulations are given along the $y$-axis on the left. The box plot of the standard error of $\widehat{\beta}_{3}$ is similar to the standard error of $\widehat{\beta}_{2}$ and omitted to save space. 
(a)
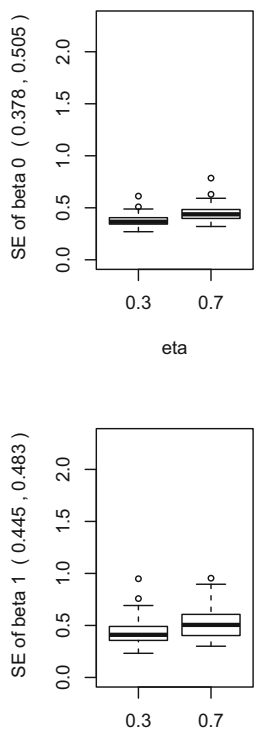

eta
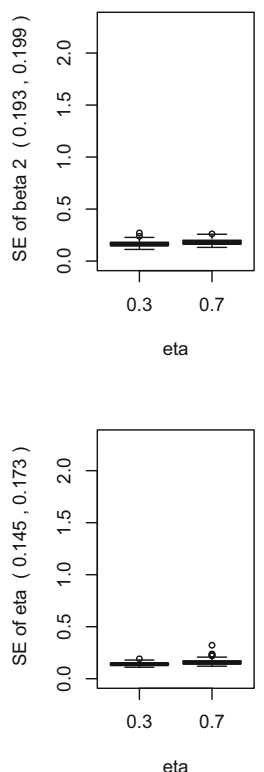

(b)
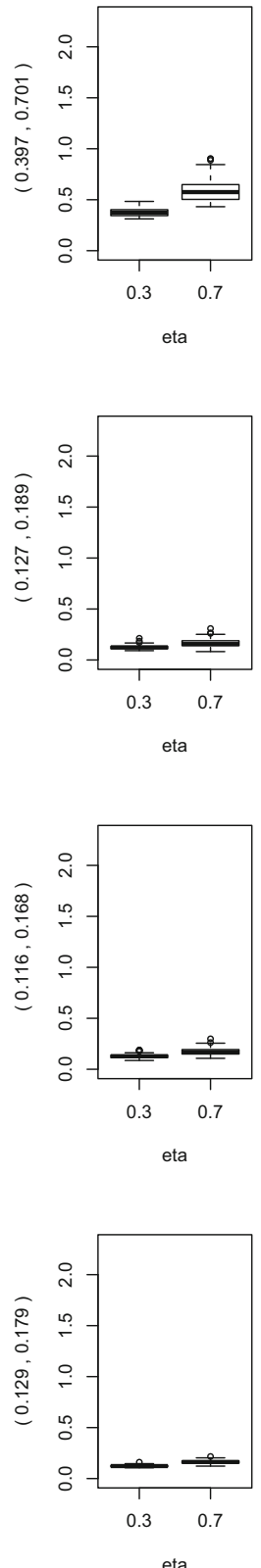

(c)
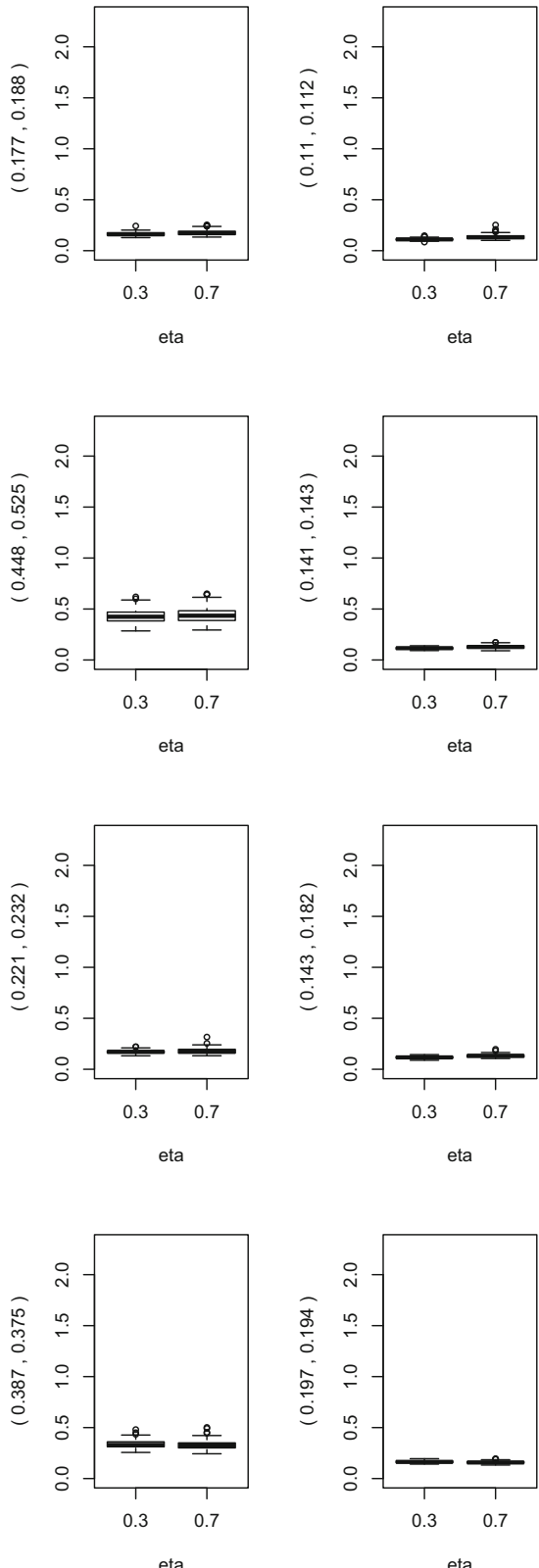

Figure 5. Box plot of the standard error of $\widehat{\beta}_{0}$ (row 1), $\widehat{\beta}_{1}$ (row 2), $\widehat{\beta}_{2}$ (row 3), and $\widehat{\eta}$ (row 4) from the 100 simulations with sample size $n=900$. Column (a): uncentered model with $\beta_{1}=5$ (weak large-scale structure); (b): uncentered model with $\beta_{1}=1$ (strong large-scale structure); (c): centered model with $\beta_{1}=5$ (weak largescale structure); (d): centered model with $\beta_{1}=1$ (strong large-scale structure). The nominal standard error of the MPPLE from the 100 simulations are given along the $y$-axis on the left. The box plot of the standard error of $\widehat{\beta_{3}}$ is similar to the standard error of $\widehat{\beta}_{2}$ and omitted to save space. 